\title{
ON THE ELEMENTARY IDEALS OF LINK MODULES
}

\author{
BY \\ R. H. CROWELL $\left({ }^{1}\right)$ AND D. STRAUSS
}

1. Introduction. Let $H$ be a free abelian multiplicative group of rank $m \geqq 1$, and let $Z(H)$ be the integral group ring of $H$. The augmentation ideal of $H$, which we denote by $I(H)$, is the kernel of the ring homomorphism $\varepsilon: Z(H) \rightarrow Z$ defined by setting $\varepsilon(h)=1$ for all $h \in H$. In this paper we shall use the expressions $H$-module and $H$-morphism as abbreviations for $Z(H)$-module and $Z(H)$-morphism, respectively. One $H$-module of interest will be the ideal $I(H)$.

An exact sequence of $H$-morphisms

$$
0 \rightarrow B \rightarrow A \rightarrow I(H) \rightarrow 0
$$

will be called a link module sequence relative to a specified basis $\left(t_{1}, \ldots, t_{m}\right)$ of $H$ if it satisfies the algebraic conditions given at the beginning of $\$ 3$. However, the motivation for the definition lies in knot theory. Consider a tame oriented link $L \subset S^{3}$ with $m$ components embedded in an oriented 3-sphere. If $m=1$, then $L$ is a knot. Let $G=\pi_{1}\left(S^{3}-L\right)$, denote the commutator subgroup by $G^{\prime}$, and set $H=G / G^{\prime}$ $=H_{1}\left(S^{3}-L\right)$. Then $H$ is free abelian of rank $m$ with a natural basis dual to $L$. The exact sequence $1 \rightarrow G^{\prime} \rightarrow G \rightarrow H \rightarrow 1$ determines, as described in [2] and [3], an exact module sequence (1), which can be shown to be a link module sequence relative to the natural basis in $H$. The module $B$ is the commutator quotient group $G^{\prime} / G^{\prime \prime}$ written additively and with the action of $H$ defined by conjugation in $G$. The module $A$ is the "Alexander module" and has a relation matrix equal to the Alexander matrix of any finite presentation of $G$.

In the sequence (1) let $E_{1}(A)$ be the 1st elementary ideal, and $\Delta_{1}$ the Alexander polynomial of the module $A$ (the definitions are given in $\$ 2$ ). Then,

$$
\Delta_{1}=\operatorname{gcd}\left(E_{1}(A)\right) \text {, }
$$

i.e., the ideal $\left(\Delta_{1}\right)$ is the smallest principal ideal containing $E_{1}(A)$. Let $E_{0}(B)$ be the 0 th ideal of the module $B$. It is a standard result of knot theory that if $m=1$, then

$$
E_{0}(B)=\left(\Delta_{1}\right)
$$

This equation was also known to be true for $m=2$, see [2, p. 297]. Milnor recently conjectured the following result, which we prove in this paper.

(1.1) THEOREM. For any link module sequence (1) and any integer $m \geqq 2$,

$$
E_{0}(B)=\Delta_{1} I(H)^{k}, \quad \text { where } k=(m-2)(m-3) / 2 .
$$

Presented to the Society, April 13, 1968; received by the editors September 20, 1968.

(1) This research was supported by the National Science Foundation (GP-8057). 
As we shall see, the ideal $I(H)$ is generated by the set of relatively prime elements $t_{1}-1, \ldots, t_{m}-1$. It is therefore a simple corollary of (1.1) that

$$
\Delta_{1}=\operatorname{gcd}\left(E_{0}(B)\right) \text {. }
$$

Compare Blanchfield's statement of this result [1, p. 340]. Thus, the Alexander polynomial of $A$ is the 0th polynomial (or elementary divisor) of $B$.

Among the tools used in proving Milnor's conjecture, we note Proposition (4.1), which is a general algebraic theorem on multilinear mappings, and, as far as we know, is new. This paper builds on the results in [2] and uses essentially the same terminology.

2. Finitely presented modules. Let $R$ be a commutative ring with 1 . If $X$ and $Y$ are free $R$-modules and if $\partial: X \rightarrow Y$ is an $R$-morphism, then by the matrix of $\partial$ relative to bases $\left(a_{i}\right)$ of $X$ and $\left(b_{j}\right)$ of $Y$ we shall mean the matrix $\left(m_{i j}\right)$ of ring elements defined by

$$
\partial\left(a_{i}\right)=\sum_{j} m_{i j} b_{j}
$$

A presentation of an $R$-module $A$, abbreviated by $c_{i} \stackrel{\partial_{2}}{\longrightarrow} b_{j} \stackrel{\partial_{1}}{\rightarrow} A$, consists of an exact sequence

$$
X_{2} \stackrel{\partial_{2}}{\longrightarrow} X_{1} \stackrel{\partial_{1}}{\longrightarrow} A \longrightarrow 0
$$

of $R$-morphisms in which $X_{1}$ and $X_{2}$ are free modules with distinguished bases $\left(b_{j}\right)$ and $\left(c_{i}\right)$, respectively. The matrix of $\partial_{2}$ relative to the bases $\left(c_{i}\right)$ and $\left(b_{j}\right)$ is the matrix of the presentation, and is also called a relation matrix for $A$. The presentation is finite if both bases $\left(c_{i}\right)$ and $\left(b_{j}\right)$ are finite, and $A$ is finitely presented if it has a finite presentation. A finite presentation in which $\left(c_{i}\right)$ and $\left(b_{j}\right)$ contain respectively $n$ and $r$ elements defines an $n \times r$ matrix and is called an $n \times r$ presentation.

Consider an arbitrary $n \times r$ matrix $M$ with entries in the ring $R$. For every nonnegative integer $k$, we define the $k$ th elementary ideal of $M$, denoted by $E_{k}(M)$, to be the ideal of $R$ generated by all $(r-k) \times(r-k)$ minor determinants of $M$. In addition, we set $E_{k}(M)=0$ if $n<r-k$, and $E_{k}(M)=R$ if $r-k \leqq 0$. It can be proved that if $M$ and $M^{\prime}$ are any two finite relation matrices of the same $R$-module, then $E_{k}(M)=E_{k}\left(M^{\prime}\right)$ for all $k=0,1,2, \ldots$ As a result, we define the $k$ th elementary ideal $E_{k}(A)$ of a finitely presented $R$-module $A$ to be the $k$ th elementary ideal of any finite relation matrix for $A$.

If $R$ is a ufd and if $A$ is finitely presented, then the $k$ th elementary divisor $\Delta_{k}$ of any $n \times r$ relation matrix is the gcd of all $(r-k) \times(r-k)$ minor determinants, with the proviso that $\Delta_{k}=0$ if $n<r-k$ and that $\Delta_{k}=1$ if $r-k \leqq 0$. It is straightforward to verify that the ideal $\left(\Delta_{k}\right)$ is the intersection of all principal ideals of $R$ which contain $E_{k}(A)$. Thus the elementary divisors are also invariants of $A$. If $R$ is the integral 
group ring of the free abelian multiplicative group $H$ with basis $\left(t_{1}, \ldots, t_{m}\right)$, then $\Delta_{k}$ is called the $k$ th polynomial of $A$, and $\Delta_{1}$ is the Alexander polynomial.

3. Link module sequences. Let $H$ be a free abelian multiplicative group of rank $m \geqq 1$. An exact sequence of $H$-morphisms

$$
0 \longrightarrow B \stackrel{\psi}{\longrightarrow} A \stackrel{\phi}{\longrightarrow} I(H) \rightarrow 0
$$

is a link module sequence relative to a basis $\left(t_{1}, \ldots, t_{m}\right)$ of $H$ if the module $A$ has an $n \times(n+1)$ presentation $c_{i} \stackrel{\partial_{2}}{\longrightarrow} b_{j} \stackrel{\partial_{1}}{\longrightarrow} A$ such that $m \leqq n+1$ and

$$
\begin{aligned}
\phi \partial_{1}\left(b_{j}\right) & =t_{j}-1, & & \text { if } 1 \leqq j \leqq m, \\
& =0, & & \text { if } m+1 \leqq j \leqq n+1 .
\end{aligned}
$$

This definition is less restrictive than the one given in [2]. The reason is simply that these conditions are sufficient to enable us to prove Theorem (1.1).

In the remainder of this section we show how to construct a finite presentation of the module $B$ in the sequence (2), given the special $n \times(n+1)$ presentation $c_{i} \stackrel{\partial_{2}}{\longrightarrow} b_{j} \stackrel{\partial_{1}}{\longrightarrow} A$. It is known [4, p. 189] that there exists an exact sequence of $H$ morphisms

$$
Y_{3} \stackrel{\alpha_{3}}{\longrightarrow} Y_{2} \stackrel{\alpha_{2}}{\longrightarrow} Y_{1} \stackrel{\alpha_{1}}{\longrightarrow} I(H) \longrightarrow 0,
$$

in which $Y_{1}, Y_{2}$, and $Y_{3}$ are free with bases

$$
\begin{array}{ll}
\left(e_{j}\right), & 1 \leqq j \leqq m, \\
\left(e_{i j}\right), & 1 \leqq i<j \leqq m, \\
\left(e_{i j k}\right), & 1 \leqq i<j<k \leqq m,
\end{array}
$$

respectively, and for which

$$
\begin{aligned}
\alpha_{1}\left(e_{j}\right) & =t_{j}-1, \\
\alpha_{2}\left(e_{i j}\right) & =\left(t_{i}-1\right) e_{j}-\left(t_{j}-1\right) e_{i}, \\
\alpha_{3}\left(e_{i j k}\right) & =\left(t_{i}-1\right) e_{j k}-\left(t_{j}-1\right) e_{i k}+\left(t_{k}-1\right) e_{i j} .
\end{aligned}
$$

If $m=1$, then $Y_{2}=Y_{3}=0$; and if $m=2$, then $Y_{3}=0$. The existence of the epimorphism $\alpha_{1}$ shows that $I(H)$ is generated by the elements $t_{1}-1, \ldots, t_{m}-1$.

The presentation $c_{i} \stackrel{\partial_{2}}{\longrightarrow} b_{j} \stackrel{\partial_{1}}{\longrightarrow} A$ consists of an exact sequence

$$
X_{2} \stackrel{\partial_{2}}{\longrightarrow} X_{1} \stackrel{\partial_{1}}{\longrightarrow} A \longrightarrow 0
$$

in which $X_{1}$ and $X_{2}$ are free with bases $\left(b_{j}\right)$ and $\left(c_{i}\right)$, respectively. Since

$$
\alpha_{1}\left(e_{j}\right)=t_{j}-1=\phi \partial_{1}\left(b_{j}\right), \quad 1 \leqq j \leqq m,
$$

we can identify $e_{j}$ and $b_{j}$ for $1 \leqq j \leqq m$. Setting $W$ equal to the free submodule of $X_{1}$ 
with basis $\left(b_{m+1}, \ldots, b_{n+1}\right)$, we obtain the following commutative diagram, in which $\pi_{1}$ is the projection onto the first summand,

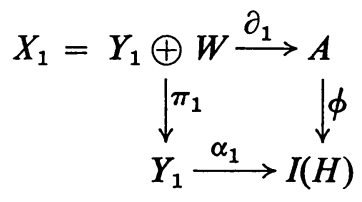

It is straightforward to verify that there exists a unique morphism $\beta_{1}$ such that $\psi \beta_{1}=\partial_{1}\left(\alpha_{2} \oplus 1_{W}\right)$, as shown in Figure 1. The morphism $1_{W}: W \rightarrow W$ is the identity. Moreover, $\beta_{1}$ is an epimorphism. One may also check that

$$
\text { Image }\left(\partial_{2}\right) \subset \text { Image }\left(\alpha_{2} \oplus 1_{w}\right),
$$

and, since $X_{2}$ is projective, there exists a (generally not unique) morphism $\beta$ such that $\partial_{2}=\left(\alpha_{2} \oplus 1_{W}\right) \beta$. Finally, therefore, we obtain the mapping diagram shown in Figure 1, in which $\beta_{2} \mid Y_{3}=\alpha_{3}$ and $\beta_{2} \mid X_{2}=\beta$. It is not difficult to show that the top row is exact, and this row therefore gives a finite presentation of the module $B$.

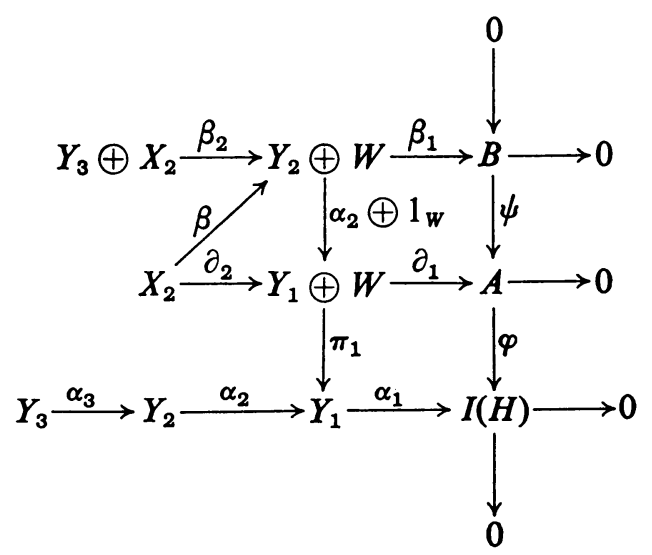

FIGURE 1

4. Multilinear mappings. The relation between the elementary ideals $E_{1}(A)$ and $E_{0}(B)$ is found by studying the matrices of the various morphisms of free modules shown in Figure 1. Before doing this, however, we prove the following general algebraic result. For any set $A$ and positive integer $n$, denote by $A^{n}$ the cartesian product of $A$ with itself $n$ times.

(4.1) Proposition. Let $R$ be a commutative ring without zero divisors, $A$ an $R$-module, and $f, g: A^{n} \rightarrow R$ two multilinear mappings such that $g\left(a_{1}, \ldots, a_{n}\right)=0$ whenever $f\left(a_{1}, \ldots, a_{n}\right)=0$. If there exist elements $r, s \in R$ and $b_{1}, \ldots, b_{n} \in A$ such that

$$
r f\left(b_{1}, \ldots, b_{n}\right)=s g\left(b_{1}, \ldots, b_{n}\right) \neq 0
$$

then $r f=s g$. 
Proof. By induction on $n$. Let $n=1$, and assume that

$$
r f(b)=s g(b) \neq 0 .
$$

Consider an arbitrary element $a \in A$. Then,

$$
f(f(a) b-f(b) a)=f(a) f(b)-f(b) f(a)=0 .
$$

Hence,

$$
0=g(f(a) b-f(b) a)=f(a) g(b)-f(b) g(a),
$$

from which we obtain $f(a) g(b)=f(b) g(a)$ and, thence, $s f(a) g(b)=s f(b) g(a)$. It follows from (4) that $r f(a) f(b)=s f(a) g(b)$. Hence, $r f(a) f(b)=s f(b) g(a)$. Since $R$ has no zero divisors, it also follows from (4) that $f(b) \neq 0$. We conclude that $r f(a)=s g(a)$.

Next, we assume that $n>1$ and that $r f\left(b_{1}, \ldots, b_{n}\right)=s g\left(b_{1}, \ldots, b_{n}\right) \neq 0$. Let $a_{1}, \ldots, a_{n}$ be arbitrary elements of $A$. It follows by induction that

$$
\begin{aligned}
& r f\left(a_{1}, b_{2}, \ldots, b_{n}\right)=s g\left(a_{1}, b_{2}, \ldots, b_{n}\right), \\
& r f\left(b_{1}, a_{2}, \ldots, a_{n}\right)=s g\left(b_{1}, a_{2}, \ldots, a_{n}\right) .
\end{aligned}
$$

Case 1. $g\left(a_{1}, b_{2}, \ldots, b_{n}\right) \neq 0$. We know that $s \neq 0$ and, consequently, that $s g\left(a_{1}, b_{2}, \ldots, b_{n}\right) \neq 0$. Hence, from (5), using induction, we obtain $r f\left(a_{1}, \ldots, a_{n}\right)$ $=\operatorname{sg}\left(a_{1}, \ldots, a_{n}\right)$.

Case 2. $g\left(a_{1}, b_{2}, \ldots, b_{n}\right)=0$. By induction we have

$$
r f\left(a_{1}+b_{1}, b_{2}, \ldots, b_{n}\right)=s g\left(a_{1}+b_{1}, b_{2}, \ldots, b_{n}\right) .
$$

But

$$
\begin{aligned}
g\left(a_{1}+b_{1}, b_{2}, \ldots, b_{n}\right) & =g\left(a_{1}, b_{2}, \ldots, b_{n}\right)+g\left(b_{1}, \ldots, b_{n}\right) \\
& =g\left(b_{1}, \ldots, b_{n}\right) \neq 0 .
\end{aligned}
$$

Consequently, we may apply induction to (7) and conclude that

$$
r f\left(a_{1}+b_{1}, a_{2}, \ldots, a_{n}\right)=\operatorname{sg}\left(a_{1}+b_{1}, a_{2}, \ldots, a_{n}\right) .
$$

Using multilinearity and equation (6), we then obtain

$$
r f\left(a_{1}, \ldots, a_{n}\right)=\operatorname{sg}\left(a_{1}, \ldots, a_{n}\right) .
$$

This completes the proof.

The essential method of relating the ideals $E_{0}(B)$ and $E_{1}(A)$ is an application of Proposition (4.1) and is described in $\S 6$. The next section contains in part rather tedious, but unfortunately necessary, details. We recommend reading the statements of the results and skipping the proofs.

5. The resolution of $I(H)$. We refer here to the exact sequence (3). The group ring $Z(H)$ of the free abelian group $H$ is an integral domain, and we may consider 
its field $F$ of fractions. If $\gamma: X \rightarrow X^{\prime}$ is any $H$-morphism, we define the rank and nullity of $\gamma$ to be the rank and nullity respectively of the linear transformation

$$
1 \otimes \gamma: F \otimes_{H} X \rightarrow F \otimes_{H} X^{\prime} .
$$

If $X$ is a free module of rank $n$, then

$$
n=\operatorname{nullity}(\gamma)+\operatorname{rank}(\gamma)
$$

If both $X$ and $X^{\prime}$ are free and if $\gamma$ is represented by an $n \times n^{\prime}$ matrix, then the rank of $\gamma$ is equal to the rank of this matrix, i.e., to the number of independent rows (or columns). Finally, if $X \stackrel{\gamma}{\rightarrow} X^{\prime} \stackrel{\gamma^{\prime}}{\rightarrow} X^{\prime \prime}$ is an exact sequence of $H$-morphisms, then the induced sequence $F \otimes_{H} X \rightarrow F \otimes_{H} X^{\prime} \rightarrow F \otimes_{H} X^{\prime \prime}$ is also exact [5, p. 170], and so the rank of $\gamma$ is equal to the nullity of $\gamma^{\prime}$.

For each of the two integers $i=2$ and 3, we denote by $N_{i}(m)$ the matrix of the morphism $\alpha_{i}$ in the sequence (3) relative to the given bases of $Y_{i}$ and $Y_{i-1}$. The notation ${ }_{j} C_{k}$ has been used throughout the paper for the combinatorial number

$$
\left(\begin{array}{l}
j \\
k
\end{array}\right)=\frac{j !}{k !(j-k) !}
$$

(5.1) The rank of $N_{2}(m)$ is equal to $m-1$, and the rank of $N_{3}(m)$ is equal to ${ }_{m-1} C_{2}$.

Proof. We may regard the morphism $\alpha_{1}$ as a mapping $\alpha_{1}: Y_{1} \rightarrow Z(H)$ between free modules. As such, it obviously has rank 1 and nullity $m-1$. Since $Y_{2} \stackrel{\alpha_{2}}{\longrightarrow} Y_{1}$ $\stackrel{\alpha_{1}}{\longrightarrow} Z(H)$ is exact,

$$
\operatorname{rank}\left(\alpha_{2}\right)=\operatorname{nullity}\left(\alpha_{1}\right)=m-1
$$

Hence,

$$
\text { nullity }\left(\alpha_{2}\right)={ }_{m} C_{2}-\operatorname{rank}\left(\alpha_{2}\right)={ }_{m} C_{2}-(m-1)={ }_{m-1} C_{2} .
$$

Since $Y_{3} \stackrel{\alpha_{3}}{\longrightarrow} Y_{2} \stackrel{\alpha_{2}}{\longrightarrow} Y_{1}$ is exact,

$$
\operatorname{rank}\left(\alpha_{3}\right)=\operatorname{nullity}\left(\alpha_{2}\right)={ }_{m-1} C_{2} \text {. }
$$

This completes the proof, since rank $N_{2}(m)=\operatorname{rank}\left(\alpha_{2}\right)$ and $\operatorname{rank} N_{3}(m)=\operatorname{rank}\left(\alpha_{3}\right)$.

As noted above, the augmentation ideal $I(H)$ is generated by the set of relatively prime elements $t_{1}-1, \ldots, t_{m}-1$. For convenience, we shall adopt the abbreviation

$$
s_{j}=t_{j}-1, \quad 1 \leqq j \leqq m .
$$

Then,

$$
\begin{aligned}
\alpha_{1}\left(e_{j}\right) & =s_{j}, \\
\alpha_{2}\left(e_{i j}\right) & =s_{i} e_{j}-s_{j} e_{i}, \\
\alpha_{3}\left(e_{i j k}\right) & =s_{i} e_{j k}-s_{j} e_{i k}+s_{k} e_{i j} .
\end{aligned}
$$

The columns of the matrix $N_{2}(m)$ are indexed by the integers $j$ with $1 \leqq j \leqq m$. The rows of $N_{2}(m)$, and likewise the columns of $N_{3}(m)$, are indexed by pairs $i j$ of integers 
with $1 \leqq i<j \leqq m$. The rows of $N_{3}(m)$ are indexed by triples $i j k$ of integers with $1 \leqq i<j<k \leqq m$.

A form of the matrix $N_{2}(m)$, particularly useful for arguments by induction on $m$, is obtained if the rows and columns are arranged as shown in Figure 2. Moreover, this form provides a simple alternative proof that rank $N_{2}(m)=m-1$. It is easy to verify that any row corresponding to a pair not containing $m$ is a linear combination, over the quotient field $F$, of the first $m-1$ rows.

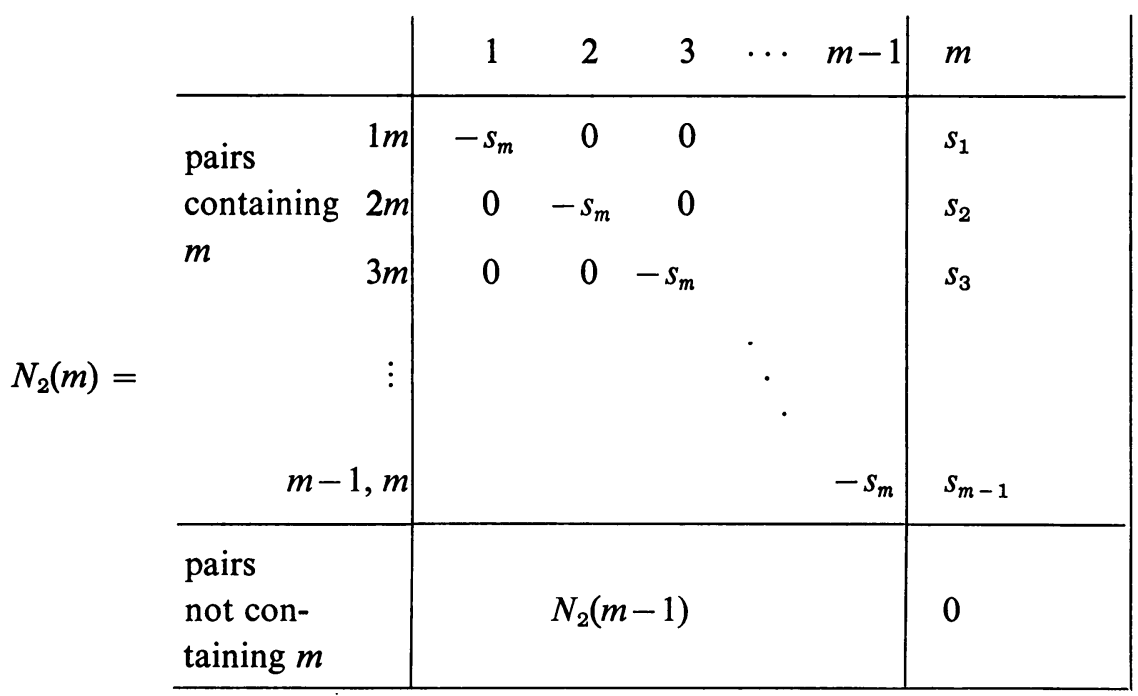

FIGURE 2

(5.2) Consider any product $u=s_{1}^{q_{1}} s_{2}^{q_{2}} \cdots s_{m}^{q_{m}}$, where $0<q_{1}+\cdots+q_{m} \leqq m-1$. If $q_{i} \geqq 1$, then $\pm u$ is equal to a minor determinant of $N_{2}(m)$ which does not contain column $i$.

Proof. By induction on $m$. For $m=2$, we have $N_{2}(2)=\left(-s_{2}, s_{1}\right)$, and the proposition is obviously true. So we assume that $m>2$ and that the statement holds for $m-1$. Next, without loss of generality, we may suppose that $i=m$. This fact is possibly not quite so obvious as it may first seem. The morphism $\alpha_{2}$ has been defined relative to an ordering of the generators $s_{1}, \ldots, s_{m}$. A reordering has the effect of permuting rows and columns of $N_{2}(m)$ and of changing the sign of some of the rows. Having disposed of this point and assuming that $i=m$, we consider two cases. First, if $q_{1}+\cdots+q_{m-1}=0$, then $u=s_{m}^{q_{m}}$ and $\pm u$ is equal to the determinant of a principal minor of the upper lefthand block of the matrix in Figure 2. This minor clearly does not involve column $m$. Second, suppose that $q_{1}+\cdots+q_{m-1}>0$. Then, since $q_{m} \geqq 1$, we have $0<q_{1}+\cdots+q_{m-1} \leqq m-2$. If we set $u^{\prime}=s_{1}^{q_{1}} s_{2}^{q_{2}} \cdots s_{m-1}^{q_{m-1}}$, then, by induction, $\pm u^{\prime}$ is equal to a minor determinant of $N_{2}(m-1)$, which may be regarded as lying in the lower lefthand block of the matrix in Figure 2. It involves $q_{1}+\cdots+q_{m-1}$ columns, leaving at least $q_{m}$ of the first $m-1$ columns uninvolved. 
By adjoining $q_{m}$ of these columns, together with the rows in which their nonzero entries occur, to the minor with determinant $\pm u^{\prime}$, we obtain a minor of $N_{2}(m)$ with determinant $\pm u$. As required, it does not involve column $m$.

We adopt the convention that the $0 \times 0$ null matrix has determinant 1 and is a minor of every matrix.

(5.3) If the product $u= \pm s_{1}^{q_{1}} \cdots s_{m}^{q_{m}}$ with $q_{1}+\cdots+q_{m} \leqq m-1$ is equal to the determinant of a minor $U$ of the matrix $N_{2}(m)$ and if $v$ is a factor of $u$, then $\pm v$ is équal to the determinant of a subminor of $U$.

Proof. The statement is obviously true for $m=2$; so we assume that $m>2$. Let $v=s_{1}^{r_{1}} \cdots s_{m}^{r_{m}}$, where $r_{1} \leqq q_{1}, \ldots, r_{m} \leqq q_{m}$. The minor $U$ contains at most $m-1$ columns of $N_{2}(m)$, and, without loss of generality, we may suppose that column $m$ is not involved. Clearly $\pm s_{m}^{q_{m}}$ is equal to the determinant of a subminor $U^{\prime}$ of $U$ which lies in the upper lefthand block of Figure 2. The factor $\pm s_{1}^{q_{1}} \cdots s_{m-1}^{q_{m-1}}$ is the determinant of a subminor $U^{\prime \prime}$ which is complementary to $U^{\prime}$ in $U$ and is a minor of $N_{2}(m-1)$. Since the rank of $N_{2}(m-1)$ is $m-2$, it follows that $q_{1}+\ldots+q_{m-1}$ $\leqq m-2$. Obviously, $\pm s_{m}^{r_{m}}$ is equal to the determinant of a subminor $V^{\prime}$ of $U^{\prime}$, and, by induction, $\pm s_{1}^{r_{1}} \cdots s_{m-1}^{r_{m-1}}$ is the determinant of a subminor $V^{\prime \prime}$ of $U^{\prime \prime}$. The subminor of $U$ containing the rows and columns of both $V^{\prime}$ and $V^{\prime \prime}$ has determinant equal to $\pm v$.

The remainder of this section is devoted to proving Proposition (5.6), which is essentially the analogue of (5.2) for the matrix $N_{3}(m)$. We recall that $N_{3}(m)$ is the ${ }_{m} C_{3} \times{ }_{m} C_{2}$ matrix of the morphism $\alpha_{3}$ and that its rank is equal to ${ }_{m-1} C_{2}$. A convenient arrangement of its rows and columns is shown in Figure 3.

\begin{tabular}{|c|c|c|c|}
\hline & & $\begin{array}{l}\text { pairs without } m \\
12\end{array}$ & $\begin{array}{c}\text { pairs with } m \\
1 m, 2 m \quad m-1, m\end{array}$ \\
\hline$N_{3}(m)=$ & $\begin{array}{l}\quad 12 m \\
\text { triples } \\
\text { with } m \\
m-2, m-1, m\end{array}$ & $\begin{array}{lllll}s_{m} & 0 & & & \\
0 & s_{m} & & \\
& & & & \\
& & \cdot & \\
& & & \cdot & \\
& & & & \\
& & & s_{m}\end{array}$ & $N_{2}(m-1)$ \\
\hline & $\begin{array}{l}\text { triples } \\
\text { without } m\end{array}$ & $N_{3}(m-1)$ & 0 \\
\hline
\end{tabular}

FiguRe 3

Let $\Sigma$ be an arbitrary ${ }_{m-1} C_{2} \times{ }_{m} C_{2}$ minor of $N_{3}(m)$ having independent rows. (We may alternatively think of $\Sigma$ as a choice of ${ }_{m-1} C_{2}$ independent rows of $N_{3}(m)$.) 
Denote by $\mathbf{N}(\Sigma)$ the set of all ${ }_{m-1} C_{2} \times{ }_{m-1} C_{2}$ minors of $\Sigma$, and by $\mathbf{N}(\hat{m})$ the set of all $(m-1) \times(m-1)$ minors of $N_{2}(m)$ which do not involve column $m$. There exists a 1-1 correspondence

$$
\tau_{\Sigma}: \mathbf{N}(\Sigma) \rightarrow \mathbf{N}(\hat{m})
$$

defined as follows: Each minor $S \in \mathbf{N}(\Sigma)$ involves ${ }_{m-1} C_{2}$ of the columns of $N_{3}(m)$ and leaves ${ }_{m} C_{2}-{ }_{m-1} C_{2}=m-1$ columns uninvolved. We define $\tau_{\Sigma}(S)$ to be the minor of $N_{2}(m)$ which omits column $m$ and involves the $m-1$ rows corresponding to those $m-1$ columns of $N_{3}(m)$ not involved in $S$.

(5.4) There exist nonzero elements $r$ and $s$ in $Z(H)$, dependent on $\Sigma$, such that, for every $S \in \mathbf{N}(\Sigma)$,

$$
r \operatorname{det}(S)= \pm s \operatorname{det} \tau_{\Sigma}(S)
$$

This lemma is derived from our general theorem on multilinear mappings, i.e., Proposition (4.1). We omit the proof at the moment, since in $\$ 6$ a somewhat more general result is proved from which (5.4) follows as a corollary.

We introduce the next lemma with the apologetic comment that it turns out to be useful in the proof of Proposition (5.6). Incidentally, if $u=s_{1}^{q_{1}} \cdots s_{m}^{q_{m}}$ is any product of nonnegative integral powers of the primes $s_{1}, \ldots, s_{m}$ in $Z(H)$, then by the order of $u$ we mean the sum $q_{1}+\cdots+q_{m}$.

(5.5) For any integer $m \geqq 3$, consider a product $u=s_{1}^{q_{1}} \cdots s_{m+1}^{q_{m+1}}$, where $q_{1}+\cdots$ $+q_{m+1} \leqq{ }_{m} C_{2}$ and, in addition,

(i) $q_{m+1}$ is maximal, i.e., $q_{1}, \ldots, q_{m} \leqq q_{m+1}$,

(ii) either $q_{1}, \ldots, q_{m}$ are all even or $q_{m}$ is odd.

If $u^{\prime}=s_{1}^{q_{1}} \cdots s_{m}^{q_{m}}$ and if $q_{1}+\cdots+q_{m}>_{m-1} C_{2}$, then we can write $u^{\prime}=u_{1} u_{2}$, where $u_{1}$ is a product of order ${ }_{m-1} C_{2}$ and $u_{2} \mid u_{1} s_{m}$.

Proof. For each $i=1, \ldots, m$, set

$$
r_{i}= \begin{cases}q_{i} / 2, & \text { if } q_{i} \text { is even, } \\ \left(q_{i}+1\right) / 2, & \text { if } q_{i} \text { is odd. }\end{cases}
$$

Case 1. All the integers $q_{1}, \ldots, q_{m}$ are even. Since $q_{1}+\cdots+q_{m}>_{m-1} C_{2} \geqq 1$, at least one of $q_{1}, \ldots, q_{m}$ is positive, from which it follows that $q_{m+1} \geqq 2$. Hence,

$$
r_{1}+\cdots+r_{m}=\frac{1}{2}\left(q_{1}+\cdots+q_{m}\right) \leqq \frac{1}{2}\left({ }_{m} C_{2}-2\right) \text {. }
$$

It is straightforward to check that $\frac{1}{2}{ }_{m} C_{2}-1 \leqq{ }_{m-1} C_{2}$ if and only if $0 \leqq m^{2}-5 m+8$ $=(m-2)(m-3)+2$. Since the last expression is always positive, we conclude that

$$
r_{1}+\cdots+r_{m} \leqq{ }_{m-1} C_{2} .
$$

Now choose integers $p_{1}, \ldots, p_{m}$ such that $r_{i} \leqq p_{i} \leqq q_{i}$ and $p_{1}+\cdots+p_{m}={ }_{m-1} C_{2}$. Clearly $q_{i}-p_{i} \geqq 0$, and we set

$$
\begin{aligned}
& u_{1}=s_{1}^{p_{1}} \cdots s_{m}^{p_{m}} \\
& u_{2}=s_{1}^{q_{1}-p_{1}} \cdots s_{m}^{q_{m}-p_{m}} .
\end{aligned}
$$


The order of $u_{1}$ is ${ }_{m-1} C_{2}$. Moreover, $q_{i}-p_{i} \leqq r_{i} \leqq p_{i}$. Hence, $u_{2} \mid u_{1}$, and this completes the proof for Case 1 .

Case 2. The integer $q_{m}$ is odd, and either $q_{m+1} \geqq 2$ or $m \geqq 4$. In this case we shall show that $r_{1}+\cdots+r_{m}-1 \leqq_{m-1} C_{2}$. To begin with,

$$
r_{1}+\cdots+r_{m} \leqq \frac{q_{1}+\cdots+q_{m}+m}{2}=\frac{1}{2}\left(q_{1}+\cdots+q_{m}\right)+\frac{m}{2} .
$$

If $q_{m+1} \geqq 2$, then $q_{1}+\cdots+q_{m} \leqq{ }_{m} C_{2}-2$. If $q_{m+1}<2$, then $m \geqq 4$ and $q_{i}<2$ for each $i=1, \ldots, m$, which implies that $q_{1}+\cdots+q_{m} \leqq m$. But $m \leqq{ }_{m} C_{2}-2$ if and only if $0 \leqq(m-4)(m+1)$, and the latter inequality holds if $m \geqq 4$. Thus, either way, we have

$$
q_{1}+\cdots+q_{m} \leqq{ }_{m} C_{2}-2
$$

Hence,

$$
\begin{aligned}
r_{1}+\cdots+r_{m} & \leqq \frac{1}{2}\left({ }_{m} C_{2}-2\right)+m / 2, \\
r_{1}+\cdots+r_{m}-1 & \leqq \frac{1}{2}{ }_{m} C_{2}-2+m / 2 .
\end{aligned}
$$

It is routine to check that $\frac{1}{2}{ }_{m} C_{2}-2+m / 2 \leqq{ }_{m-1} C_{2}$ if and only if $(m-4)(m-3) \geqq 0$, and the latter inequality holds for every integer $m$. So

$$
r_{1}+\cdots+r_{m}-1 \leqq{ }_{m-1} C_{2} .
$$

Choose integers $p_{1}, \ldots, p_{m}$ such that $r_{i} \leqq p_{i} \leqq q_{i}$, for $i=1, \ldots, m-1$, while $r_{m}-1$ $\leqq p_{m} \leqq q_{m}$, and $p_{1}+\cdots+p_{m}={ }_{m-1} C_{2}$. Set

$$
u_{1}=s_{1}^{p_{1}} \cdots s_{m}^{p_{m}}, \quad u_{2}=s_{1}^{q_{1}-p_{1}} \cdots s_{m}^{q_{m}-p_{m}} .
$$

For each $i=1, \ldots, m-1$, we have $q_{i}-p_{i} \leqq r_{i} \leqq p_{i}$, whereas $q_{m}-p_{m} \leqq r_{m} \leqq p_{m}+1$. It follows that $u_{2} \mid u_{1} s_{m}$, and the proof of Case 2 is completed.

Case 3. $q_{m}$ is odd, $q_{m+1}<2$, and $m=3$. In this case, $q_{4}=q_{3}=1$. Since $q_{1}+q_{2}+q_{3}$ $>_{3-1} C_{2}=1$, we know that $q_{1}+q_{2} \geqq 1$. On the other hand, $q_{1}+\cdots+q_{4} \leqq{ }_{3} C_{2}=3$; hence $q_{1}+q_{2} \leqq 1$. It follows that one of $q_{1}$ and $q_{2}$ is 0 and the other is 1 . Suppose that $q_{1}=0$ and $q_{2}=1$. Then $u=s_{2} s_{3} s_{4}$ and $u^{\prime}=s_{2} s_{3}$. If we set $u_{1}=s_{2}$ and $u_{2}=s_{3}$, then $u_{1}$ has order $1={ }_{3-1} C_{2}$ and $u_{2} \mid u_{1} s_{3}$. The argument is the same if $q_{1}=1$ and $q_{2}=0$. So this completes the proof of Case 3, and also, of Lemma (5.5).

As an aid in reading the proof of Proposition (5.6), the matrix $N_{3}(m+1)$ is illustrated in Figure 4. It is, of course, entirely analogous to $N_{3}(m)$ as shown in Figure 3.

(5.6) Proposition. If $u=s_{1}^{q_{1}} \cdots s_{m+1}^{q_{m+1}}$ is any product in which $q_{1}+\cdots+q_{m+1}$ $\leqq_{m} C_{2}$, then $\pm u$ is equal to a minor determinant of $N_{3}(m+1)$.

Proof. The proof, which is by induction on $m$, begins with $m=2$. In this case $N_{3}(m+1)=N_{3}(3)$ is the $1 \times 3$ matrix

\begin{tabular}{c|ccc} 
& 12 & 13 & 23 \\
\hline 123 & $s_{3}$ & $-s_{2}$ & $s_{1}$
\end{tabular}




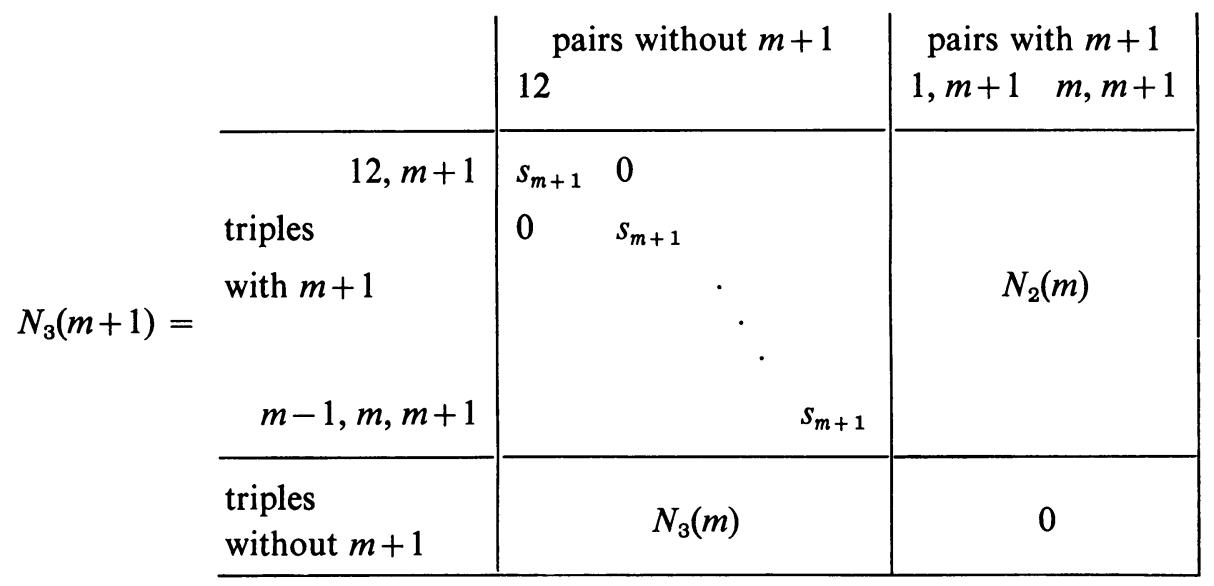

FIGURE 4

and (5.6) is obviously true. We henceforth assume that $m \geqq 3$ and make the inductive assumption that the statement of the proposition holds for $2, \ldots, m-1$.

We next establish two important observations. The first is that, if a product $w=s_{1}^{q_{1}} \cdots s_{m}^{q_{m}}$ satisfies $q_{1}+\cdots+q_{m} \leqq{ }_{m-1} C_{2}$ and if $q_{m} \geqq m-2$, then $\pm w$ is equal to the determinant of a minor of $N_{3}(m)$ which does not involve any column of the form im. This is certainly true for $m=3$, as can be seen from (8). So we shall assume that $m \geqq 4$. Since ${ }_{m-1} C_{2}-(m-2)={ }_{m-2} C_{2}$, we have $q_{1}+\cdots+q_{m-1} \leqq{ }_{m-2} C_{2}$. The inductive assumption implies that (5.6) holds with $m$ replaced by $m-2$. We conclude that $\pm s_{1}^{q_{1}} \cdots s_{m-1}^{q_{m-1}}$ is equal to the determinant of a minor of $N_{3}(m-1)$, which may be regarded as lying in the lower lefthand block of the matrix in Figure 3. By adjoining $q_{m}$ of the first ${ }_{m-1} C_{2}$ columns of this matrix, together with the rows in which their nonzero entries $s_{m}$ occur, we can clearly produce a minor of $N_{3}(m)$ with determinant $\pm w$ which does not involve any column im.

The second observation is less trivial than it first appears. Namely, if Proposition (5.6) holds for the natural ordering $1<2<\cdots<m+1$, then it also holds for any ordering of the indices. To show this, we suggest considering the effect on the matrix $N_{3}(m)$ of interchanging the order of $i$ and $i+1$. Ignoring permutations of rows and columns, we find that a given entry changes sign if and only if both $i$ and $i+1$ occur in its row label and either $i$ or $i+1$, but not both, occur in its column label. Thus, the total sign change can be accomplished by changing the sign of every row containing both $i$ and $i+1$ in its label and then changing the sign of the one column containing both $i$ and $i+1$.

Using the inductive assumption, we now prove (5.6). As a result of the second observation above, it may be supposed that the indices are ordered so that $q_{m+1}$ is maximal, and so that $q_{m}$ is odd unless $q_{1}, \ldots, q_{m}$ are all even. It may also be assumed that $q_{m+1} \geqq 1$, since otherwise $u=1$. We set $u^{\prime}=s_{1}^{q_{1}} \cdots s_{m}^{q_{m}}$.

Case 1. $q_{1}+\cdots+q_{m} \leqq_{m-1} C_{2}$. Then, by induction, $\pm u^{\prime}$ is equal to the determinant of a minor of $N_{3}(m)$, which we may regard as lying in the lower lefthand block 
of Figure 4. Since $q_{1}+\cdots+q_{m}+q_{m+1} \leqq{ }_{m} C_{2}$, there are at least $q_{m+1}$ of the first ${ }_{m} C_{2}$ columns of $N_{3}(m+1)$ which do not involve the columns of this minor. By adjoining $q_{m+1}$ of these columns, together with the rows in which their nonzero entries $s_{m+1}$ occur, we construct a minor of $N_{3}(m+1)$ with determinant $\pm u$.

Case 2. $q_{1}+\cdots+q_{m}>_{m-1} C_{2}$. Using Lemma (5.5), we write $u^{\prime}=u_{1} u_{2}$, where $u_{1}$ is a product of order ${ }_{m-1} C_{2}$ and $u_{2} \mid u_{1} s_{m}$. Since

we have

$$
\operatorname{order}\left(u^{\prime}\right) \leqq{ }_{m} C_{2}-q_{m+1} \leqq{ }_{m} C_{2}-1,
$$

$$
\operatorname{order}\left(u_{2}\right) \leqq{ }_{m} C_{2}-1-{ }_{m-1} C_{2}=m-2 .
$$

Let $u_{1} s_{m}=u_{2} v$. Then,

$$
\operatorname{order}\left(u_{2}\right)+\operatorname{order}(v)={ }_{m-1} C_{2}+1,
$$

and it is easy to check that ${ }_{m-1} C_{2}+1 \geqq m-1$ if and only if $(m-3)(m-2) \geqq 0$. Hence,

$$
\operatorname{order}\left(u_{2}\right)+\operatorname{order}(v) \geqq m-1 \text {. }
$$

It follows that there exists a nontrivial factor $v^{\prime}$ of $v$ such that order $\left(u_{2} v^{\prime}\right)=m-1$. Moreover, since $v^{\prime}$ is nontrivial and since either $u_{2}$ or $v$ has $s_{m}$ as a factor, we may select $v^{\prime}$ so that the product $u_{2} v^{\prime}$ contains the factor $s_{m}$. We set

Clearly

$$
w=\left(v / v^{\prime}\right) s_{m}^{m-2}=\left(u_{1} s_{m} / u_{2} v^{\prime}\right) s_{m}^{m-2} .
$$

$$
\operatorname{order}(w)=\operatorname{order}\left(u_{1}\right)={ }_{m-1} C_{2} .
$$

Since $s_{m}^{m-2} \mid w$, it is a consequence of the first observation above that there exists a minor $S$ of $N_{3}(m)$ such that $\operatorname{det}(S)= \pm w$ and which does not involve any column of the form im. Let $\Sigma$ be the ${ }_{m-1} C_{2} \times{ }_{m} C_{2}$ minor of $N_{3}(m)$ of which $S$ is a subminor. From the definition of the correspondence $\tau_{\Sigma}$, it follows that $\tau_{\Sigma}(S)$ is the diagonal minor of $N_{2}(m)$ which forms the upper lefthand block in Figure 2. Hence,

$$
\operatorname{det} \tau_{\Sigma}(S)= \pm s_{m}^{m-1} .
$$

By Lemma (5.4), there exist nonzero elements $r$ and $s$ in $Z(H)$ (dependent on $\Sigma$ ) such that $r \operatorname{det}(S)= \pm s \operatorname{det} \tau_{\Sigma}(S)$, that is,

$$
r w= \pm s s_{m}^{m-1} \text {. }
$$

Since order $\left(u_{2} v^{\prime}\right)=m-1$ and since $u_{2} v^{\prime}$ contains the factor $s_{m}$, it is a corollary of Lemma (5.2) that there exists a minor $U$ of $N_{2}(m)$ with $\operatorname{det}(U)= \pm u_{2} v^{\prime}$ and which does not involve column $m$. Let $V$ be the minor of $N_{3}(m)$ such that $\tau_{\Sigma}(V)=U$. Then,

$$
r \operatorname{det}(V)= \pm s \operatorname{det}(U)= \pm s u_{2} v^{\prime}
$$

Hence, from (9) and the definition of $w$, we obtain

$$
\operatorname{det}(V)= \pm\left(w / s_{m}^{m-1}\right) u_{2} v^{\prime}= \pm\left(u_{1} s_{m} s_{m}^{m-2} / u_{2} v^{\prime} s_{m}^{m-1}\right) u_{2} v^{\prime}= \pm u_{1} \text {. }
$$


Next, using Lemma (5.3), we choose a subminor $U^{\prime}$ of $U$ with the property that $\operatorname{det}\left(U^{\prime}\right)= \pm u_{2}$.

The remainder of the proof of Case 2 is quite simple. The minor $V$ lies in the lower lefthand block of Figure 4, while $U$ lies in the upper righthand block. Since $U=\tau_{\Sigma}(V)$, the definition of $\tau_{\Sigma}$ implies that any column of $V$ and row of $U$, when extended to $N_{3}(m+1)$, must intersect in the entry 0 . Moreover, since $q_{1}+\cdots$ $+q_{m} \geqq_{m-1} C_{2}$, we know that $q_{m+1} \leqq m-1$. Hence, we may choose $q_{m+1}$ additional columns (i.e., not involved in $V$ ) from the first ${ }_{m} C_{2}$ columns of $N_{3}(m+1)$ to finally produce a minor $Q$ with the structure shown in Figure 5. The determinant of $Q$ will be evaluated using the general Laplace expansion [6, p. 112]. For this purpose, we divide $Q$ horizontally into two minors $Q_{1}$ and $Q_{2}$, where $Q_{1}$ consists of the top six blocks and $Q_{2}$ consists of the bottom three. Then, any ${ }_{m-1} C_{2} \times{ }_{m-1} C_{2}$ subminor of $Q_{2}$ either is $V$, or contains a column

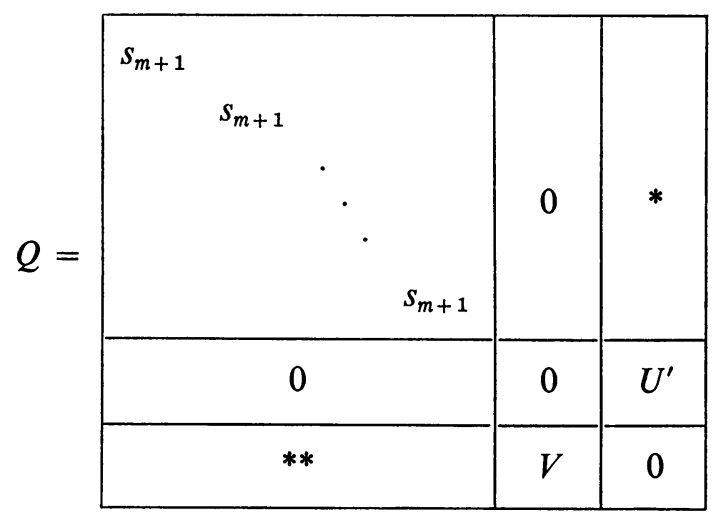

FIGURE 5

of zeros, or has a cofactor with a column of zeros. We conclude that

$$
\begin{aligned}
\operatorname{det}(Q) & = \pm \operatorname{det}(V) s_{m+1}^{q_{m+1}} \operatorname{det}\left(U^{\prime}\right) \\
& = \pm u_{1} u_{2} s_{m+1}^{q_{m+1}}= \pm u
\end{aligned}
$$

This completes the proof of Case 2, and also of Proposition (5.6).

6. Proof of Theorem (1.1). We return to Figure 1 and consideration of the matrices of morphisms of free modules shown there. All matrices will be chosen relative to the bases discussed in $\S 3$. Let $M$ be the matrix of the original $n \times(n+1)$ presentation of the module $A$. Thus, $M=$ matrix $\left(\partial_{2}\right)$, and we write $M=\left(\begin{array}{ll}M_{1} & M_{2}\end{array}\right)$, where $M_{1}$ consists of the first $m$ columns and $M_{2}$ of the last $n+1-m$. Then matrix $(\beta)$ is an $n \times\left({ }_{m} C_{2}+n+1-m\right)$ matrix of the form $\left(M^{\prime} M_{2}\right)$. Set

$$
N=\left(\begin{array}{cc}
N_{2} & 0 \\
0 & I
\end{array}\right),
$$


where $N_{2}=N_{2}(m)$ is the matrix of $\alpha_{2}$ and $I$ is the $(n+1-m) \times(n+1-m)$ identity matrix. Since $\left(\alpha_{2} \oplus 1_{W}\right) \beta=\partial_{2}$, it follows that

$$
\left(\begin{array}{ll}
M^{\prime} & M_{2}
\end{array}\right) N=\left(\begin{array}{ll}
M^{\prime} N_{2} & M_{2}
\end{array}\right)=\left(\begin{array}{ll}
M_{1} & M_{2}
\end{array}\right)=M .
$$

The matrix of $\beta_{2}$, which we denote by $P$, is the $\left(n+{ }_{m} C_{3}\right) \times\left({ }_{m} C_{2}+n+1-m\right)$ matrix

$$
P=\left(\begin{array}{cc}
M^{\prime} & M_{2} \\
N_{3} & 0
\end{array}\right)
$$

where $N_{3}=N_{3}(m)$ is the matrix of $\alpha_{3}$. Since $\alpha_{2} \alpha_{3}=0$, we have $N_{3} N_{2}=0$, and so

$$
P N=\left(\begin{array}{cc}
M^{\prime} N_{2} & M_{2} \\
N_{3} N_{2} & 0
\end{array}\right)=\left(\begin{array}{cc}
M_{1} & M_{2} \\
0 & 0
\end{array}\right)=\left(\begin{array}{c}
M \\
0
\end{array}\right) .
$$

In order to compute the elementary ideal $E_{0}(B)$, we consider the determinants of all square minors of $P$ which involve all ${ }_{m} C_{2}+n+1-m$ columns of $P$. Since

$$
{ }_{m} C_{2}+n+1-m={ }_{m} C_{2}-(m-1)+n={ }_{m-1} C_{2}+n
$$

and since, as was shown in (5.1), the rank of $N_{3}$ is ${ }_{m-1} C_{2}$, it follows that any such minor will have a nonzero determinant only if it involves precisely ${ }_{m-1} C_{2}$ independent rows of $N_{3}$.

Thus, we again consider maximal independent sets of rows of $N_{3}$. Let $q$ be an arbitrary integer such that $q \geqq m-1$. In the application to the present situation of the construction which follows, we shall let $q=n$. For the proof of Lemma (5.4), we shall take $q=m-1$. Consider the free $Z(H)$-module

$$
D=(Z(H))^{m C_{2}+q-(m-1)},
$$

whose elements will be considered as tuples, or row matrices, with entries in $Z(H)$. Let $\Sigma$ be any ${ }_{m-1} C_{2} \times\left({ }_{m} C_{2}+q-(m-1)\right)$ minor of the matrix

\begin{tabular}{c|c|c} 
& ${ }_{m} C_{2}$ & $q-(m-1)$ \\
\hline${ }_{m} C_{3}$ & $N_{3}$ & 0
\end{tabular}

having independent rows, which we denote by $\sigma_{1}, \ldots, \sigma_{m-1} C_{2}$. For each $\Sigma$, we define two multilinear mappings $f, g: D^{q} \rightarrow Z(H)$ as follows: For any $d_{1}, \ldots, d_{q} \in D$, the matrix

$$
T=\left(\begin{array}{c}
d_{1} \\
\vdots \\
d_{q} \\
\Sigma
\end{array}\right)
$$

has $q+{ }_{m-1} C_{2}$ rows and ${ }_{m} C_{2}+q-(m-1)$ columns and is therefore square. We set $f\left(d_{1}, \ldots, d_{q}\right)=\operatorname{det}(T)$. Let $I$ be the $(q-(m-1)) \times(q-(m-1))$ identity matrix, and set

$$
N=\left(\begin{array}{cc}
N_{2} & 0 \\
0 & I
\end{array}\right)
$$


The product $T N$ is defined, and, since $N_{3} N_{2}=0$, we have $\sigma_{i} N=0$. Hence,

$$
T N=\left(\begin{array}{c}
d_{1} N \\
\vdots \\
d_{q} N \\
\hline \sigma_{1} N \\
\vdots \\
\sigma_{m-1} C_{2} N
\end{array}\right)=\left(\begin{array}{c}
d_{1} N \\
\vdots \\
d_{q} N \\
\hline 0 \\
\vdots \\
0
\end{array}\right)
$$

The matrix $T N$ has $m+(q-(m-1))=q+1$ columns, the first $m$ of which correspond to the columns of $N_{2}$. We define $g\left(d_{1}, \ldots, d_{q}\right)$ to be the determinant of the minor of $T N$ obtained by deleting the $m$ th column and the last ${ }_{m-1} C_{2}$ rows, which are zero.

$$
\text { If } f\left(d_{1}, \ldots, d_{q}\right)=0 \text {, then } g\left(d_{1}, \ldots, d_{q}\right)=0 \text {. }
$$

Proof. Since $\operatorname{det}(T)=0$, there exist elements $u_{1}, \ldots, u_{q}, v_{1}, \ldots, v_{m-1} c_{2}$ in $Z(H)$ which are not all zero and for which

$$
0=u_{1} d_{1}+\cdots+u_{q} d_{q}+v_{1} \sigma_{1}+\cdots+v_{m-1} C_{2} \sigma_{m-1} C_{2} .
$$

Since $\sigma_{1}, \ldots, \sigma_{m-1} c_{2}$ are independent, we know that $u_{1}, \ldots, u_{q}$ are not all zero. Multiplying by $N$ and remembering that $\sigma_{i} N=0$, we obtain $0=u_{1} d_{1} N+\cdots+u_{q} d_{q} N$. Hence, rank $(T N)<q$, and this implies that $g\left(d_{1}, \ldots, d_{q}\right)=0$.

There exist elements $d_{1}, \ldots, d_{q} \in D$ such that $g\left(d_{1}, \ldots, d_{q}\right) \neq 0$.

Proof. The independent set $\left\{\sigma_{1}, \ldots, \sigma_{m-1} C_{2}\right\}$ of rows of $\Sigma$ can be extended to an independent set $\left\{d_{1}, \ldots, d_{q}, \sigma_{1}, \ldots, \sigma_{m-1} C_{2}\right\}$ by embedding $D$ in the vector space $F \otimes_{H} D$, where $F$ is the field of fractions of $Z(H)$. With these rows, the matrix $T$ is nonsingular. Let $N_{2}^{\prime}$ be the matrix obtained by deleting the $m$ th column of $N_{2}$. It is a consequence of Lemma (5.2) that rank $\left(N_{2}^{\prime}\right)=m-1$. If

$$
N^{\prime}=\left(\begin{array}{cc}
N_{2}^{\prime} & 0 \\
0 & I
\end{array}\right)
$$

then obviously $\operatorname{rank}\left(N^{\prime}\right)=(m-1)+q-(m-1)=q$. Since $T$ is nonsingular, it follows that rank $\left(T N^{\prime}\right)=q$, which implies that $g\left(d_{1}, \ldots, d_{q}\right) \neq 0$.

It is a simple corollary of Lemmas (6.1) and (6.2) and of Proposition (4.1) that

$$
\text { There exist nonzero elements } r \text { and } s \text { in } Z(H) \text { such that } r f=s g \text {. }
$$

We now prove Lemma (5.4). In the preceding discussion of the mappings $f, g: D^{q} \rightarrow Z(H)$, take $q=m-1$. Let $S$ be an arbitrary ${ }_{m-1} C_{2} \times{ }_{m-1} C_{2}$ subminor of $\Sigma$. We choose $d_{1}, \ldots, d_{m-1}$ as follows: Each is an ${ }_{m} C_{2}$-tuple with one entry equal to 1 and the rest zeros. The nonzero entries occur in those columns $i j$ of $N_{3}$ not involved in $S$, and there are exactly $m-1$ of these. If chosen properly, it is obvious that $f\left(d_{1}, \ldots, d_{m-1}\right)= \pm \operatorname{det}(S)$. Since $q=m-1$, we have $N=N_{2}$. The first 
$m-1$ rows of the matrix $T N=T N_{2}$, which are the only nonzero rows, are precisely those rows of $N_{2}$ corresponding to columns of $N_{3}$ not involved in $S$. Hence,

$$
g\left(d_{1}, \ldots, d_{m-1}\right)= \pm \operatorname{det} \tau_{\Sigma}(S),
$$

and (5.4) follows as a consequence of (6.3).

For the proof of Theorem (1.1), we now apply (6.3), setting $q=n$. In order to find a relation between $r$ and $s$, we substitute particular elements $d_{1}, \ldots, d_{n} \in D$ into the equation $r f=s g$. As in the preceding paragraph, each $d_{i}$ is taken to be a unit vector with one entry equal to 1 and the rest equal to 0 . The entries of $d_{i}$, or equivalently, the columns of $T$, are indexed first by the ${ }_{m} C_{2}$ pairs $j k$ with $1 \leqq j<k \leqq m$, and then by the integers $l$ with $m+1 \leqq l \leqq n+1$. An entry of $d_{i}$ will therefore be denoted by either $\left(d_{i}\right)_{j k}$ or $\left(d_{i}\right)_{l}$. The single nonzero entry of $d_{i}$ is now defined by

$$
\begin{array}{ll}
\left(d_{i}\right)_{i m}=1, & \text { if } 1 \leqq i \leqq m-1, \\
\left(d_{i}\right)_{i+1}=1, & \text { if } m \leqq i \leqq n .
\end{array}
$$

With this choice, $\pm f\left(d_{1}, \ldots, d_{n}\right)= \pm \operatorname{det}(T)$ is equal to the determinant of that minor of $\Sigma$ whose columns $j k$ satisfy $j<k<m$. That is, they are the first ${ }_{m-1} C_{2}$ columns of the matrix $N_{3}(m)$ shown in Figure 3. Since the rank of $N_{3}(m-1)$ is ${ }_{m-2} C_{2}$, at most ${ }_{m-2} C_{2}$ of the rows of the bottom two blocks of Figure 3 are independent. Thus, at least ${ }_{m-1} C_{2}-{ }_{m-2} C_{2}=m-2$ among the rows $\sigma_{1}, \ldots, \sigma_{m-1} C_{2}$ of $\Sigma$ occur in the upper portion of Figure 3. It follows that

$$
f\left(d_{1}, \ldots, d_{n}\right)= \pm s_{m}^{m-2} u
$$

where $u$ is a homogeneous polynomial in $s_{1}, \ldots, s_{m}$ of order equal to

$$
{ }_{m-1} C_{2}-(m-2)={ }_{m-2} C_{2}=(m-2)(m-3) / 2 .
$$

For this particular set of elements $d_{1}, \ldots, d_{n}$, the nonzero rows of $T N$ are either the rows im in $N_{2}(m)$, as shown in Figure 2, or are unit vectors with nonzero entries among the coordinates $m+1, \ldots, n+1$. Thus, $\pm g\left(d_{1}, \ldots, d_{n}\right)$ is the determinant of a matrix of the form shown in Figure 6. Hence, $g\left(d_{1}, \ldots, d_{n}\right)= \pm s_{m}^{m-1}$. Since $r f=s g$, we obtain as a fraction

$$
\frac{s}{r}= \pm \frac{s_{m}^{m-2} u}{s_{m}^{m-1}}= \pm \frac{u}{s_{m}}
$$

To get a proof of (1.1), take for $d_{1}, \ldots, d_{n}$ the rows of the matrix $\left(\begin{array}{ll}M^{\prime} & M_{2}\end{array}\right)$ described in the first paragraph of this section. In this case $f\left(d_{1}, \ldots, d_{n}\right)$ is one of the generators of $E_{0}(B)$. Moreover, $T$ is a submatrix of $P$, and

$$
T N=\left(\begin{array}{c}
M \\
0
\end{array}\right)
$$

It follows from Proposition (3.1) in [2, p. 292] that $g\left(d_{1}, \ldots, d_{n}\right)= \pm \Delta_{1} s_{m}$, where $\Delta_{1}$ is the Alexander polynomial of the matrix $M$, and hence, of the module $A$. 


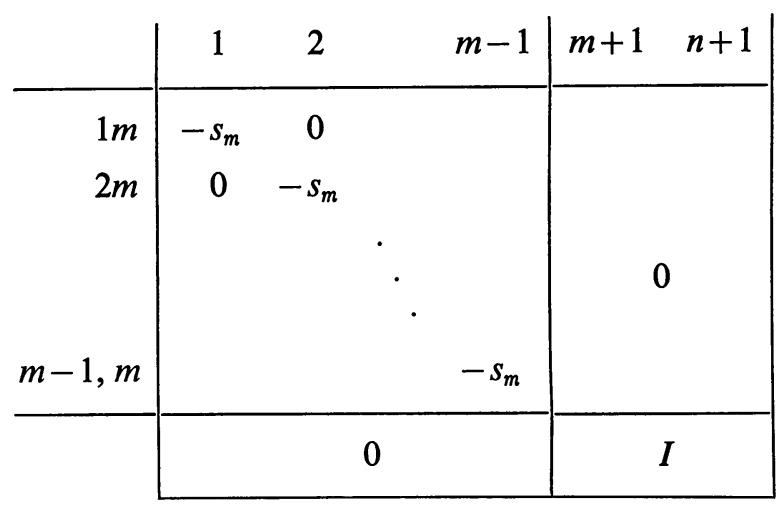

FIGURE 6

From the equation $r f=s g$ and (10), we have

$$
f\left(d_{1}, \ldots, d_{n}\right)= \pm \frac{u}{s_{m}} \Delta_{1} s_{m}= \pm u \Delta_{1} .
$$

A complete set of generators of $E_{0}(B)$ is obtained by taking all possible choices of $\Sigma$, i.e., all possible sets of ${ }_{m-1} C_{2}$ independent rows of $N_{3}$. Consider an arbitrary product $u=s_{1}^{q_{1}} \cdots s_{m}^{q_{m}}$ with $q_{1}+\cdots+q_{m}={ }_{m-2} C_{2}$. We contend that $\Sigma$ can be chosen so that this $u$ occurs in (11). If $m=2$ or 3 , then necessarily $u=1$, so we may assume $m \geqq 4$. Let $u^{\prime}=s_{1}^{q_{1}} \cdots s_{m-1}^{q_{m-1}}$. Then $q_{1}+\cdots+q_{m-1} \leqq_{m-2} C_{2}$ and, by Proposition (5.6), $\pm u^{\prime}$ is equal to the determinant of a minor of $N_{3}(m-1)$, which we regard as lying in the lower lefthand block of Figure 3. By adjoining the remaining columns of the lefthand side of $N_{3}(m)$, i.e., columns $i j$ which do not involve this minor and for which $i<j<m$, together with the rows in which their entries $s_{m}$ occur, we construct a minor $Q$ of $N_{3}$. Since

$$
{ }_{m-1} C_{2}-\left(q_{1}+\cdots+q_{m-1}\right)=(m-2)+{ }_{m-2} C_{2}-\left(q_{1}+\cdots+q_{m-1}\right)=m-2+q_{m},
$$

we know that $\operatorname{det}(Q)= \pm u^{\prime} s_{m}^{q_{m}+m-2}= \pm u s_{m}^{m-2}$, and this establishes our contention. It then follows from (11) that

$$
E_{0}(B)=I(H)^{k} \Delta_{1}, \quad \text { where } k=(m-2)(m-3) / 2,
$$

and Theorem (1.1) is proved.

\section{REFERENCES}

1. R. C. Blanchfield, Intersection theory of manifolds with operators with applications to knot theory, Ann. of Math. 65 (1957), 340-356.

2. R. H. Crowell, Torsion in link modules, J. Math. Mech. 14 (1965), 289-298.

3. - Corresponding group and module sequences, Nagoya Math. J. 19 (1961), 27-40.

4. S. MacLane, Homology, Academic Press, New York, 1963.

5. D. G. Northcott, An introduction to homological algebra, Cambridge Univ. Press, Cambridge, 1960.

6. O. Schreier and E. Sperner, Modern algebra and matrix theory, Chelsea, New York, 1955.

Dartmouth College,

HANOVER, New HampShiRe 